\title{
Valorización de la glicerina mediante el Procesado Acuoso a Presión (PAP): influencia del 25\% de Fe en el catalizador Ni/Al
}

\author{
Raquel Raso, Joaquín Ruíz, Miriam Oliva, Lucía García, Jesús Arauzo \\ Grupo de Procesos Termoquímicos (GPT) \\ Instituto de Investigación en Ingeniería de Aragón (I3A) \\ Universidad de Zaragoza, Mariano Esquillor s/n, 50018, Zaragoza, Spain.
}

Tel.+34-976762707, e-mail: rroka@unizar.es

\section{Resumen}

En este trabajo se estudia el procesado acuoso a presión de la glicerina (subproducto de biodiésel) sin aporte externo de $\mathrm{H}_{2}$ para la obtención de productos de valor añadido como, por ejemplo, 1,2propanodiol. De forma específica, se ha estudiado sobre la base de un catalizador $\mathrm{Ni}-\mathrm{Al}$, la influencia de incorporar un 25\% de Fe en su estructura a efectos de la distribución de productos.

\section{Introducción}

Desde hace más de dos décadas, los biocombustibles, como por ejemplo el biodiésel, se han incorporado a los combustibles de transporte, debido a sus ventajas medioambientales y a que su uso conlleva una menor dependencia del petróleo. Actualmente, la elevada producción de biodiésel origina un exceso de glicerina generando un excedente cuando se consideran sus usos tradicionales, ya que por cada 10 toneladas de biodiésel producido se genera alrededor de 1 tonelada de glicerina. Es por ello por lo que en la última década se han estudiado y desarrollado nuevas alternativas para valorizar la glicerina que se ha generado como residuo. El aprovechamiento de la glicerina presenta ventajas, por un lado, los beneficios de su valorización contribuyen a que la producción de biodiésel sea más competitiva y por otro lado se evita la generación de un residuo y su necesario tratamiento [1-3].

En este contexto de biorrefinería, se estudia la conversión de glicerina en productos químicos, entre ellos puede citarse el 1,2-propanodiol, mediante el Procesado Acuoso a Presión (PAP). PAP es un proceso catalítico que se realiza a presiones moderadas de alrededor de 40 bar y temperaturas relativamente bajas, de alrededor de $500 \mathrm{~K}$, lo que permite la producción de gases y líquidos a partir de una materia prima renovable [3-4]. Es un proceso que no necesita de un aporte externo de hidrógeno, por lo que es menos costoso y más seguro que la hidrogenólisis convencional que sí requiere su aporte.

El objetivo de este trabajo es desarrollar un catalizador para usarlo en el PAP con glicerina, que sea estable en las condiciones de operación establecidas, ya que la estabilidad de un catalizador es muy importante para su aplicación industrial. En estudios previos con catalizadores de $\mathrm{Ni}$, se ha encontrado la formación de nuevas fases en el sólido como, por ejemplo, boehmita en catalizadores $\mathrm{Ni}-\mathrm{Al}$ por la hidratación del $\mathrm{Al}_{2} \mathrm{O}_{3}$ [4]. En este trabajo se pretende estudiar el comportamiento del catalizador $\mathrm{Ni}$-Al y ver la influencia de incorporar un $25 \%$ Fe en su estructura.

\section{Experimental}

La reacción se llevó a cabo en un reactor de lecho fijo de acero inoxidable con los catalizadores basados en níquel, Ni-Al (Fe0) y Ni-Al-Fe (Fe25). Éstos se estudiaron en una instalación a pequeña escala de laboratorio que opera en continuo, diseñada y desarrollada por PID Eng \& Tech (España). Para la preparación de estos catalizadores se ha empleado el método de co-precipitación cambiando la relación $\mathrm{Fe} / \mathrm{Al}$ (0 y 25\%). Tras ello se caracterizaron los catalizadores por diversas técnicas (BET, XRD, TPR y ICP-OES) para obtener una información detalla de los sólidos. Estos catalizadores se probaron durante 3 $\mathrm{h}$ en experimentos de PAP utilizando como reactivo una disolución de glicerina en agua al 10\% en peso con el fin de estudiar el efecto de los catalizadores sobre la conversión de glicerina y la distribución de los productos obtenidos. En la Tabla 1 se detallan los valores de temperatura $(\mathrm{T})$, presión $(\mathrm{P})$, cantidad de glicerina alimentada (F) y la relación masa de catalizador/caudal másico de compuesto orgánico alimentado $(\mathrm{W} / \mathrm{m})$. La corriente de gas fue analizada en línea por un micro-GC, equipado con detectores de conductividad térmica (TCD) que permite cuantificar los gases generados. Los productos líquidos obtenidos fueron analizados por un GC/FID 
y un analizador TOC. En intervalos de tiempo de $1 \mathrm{~h}$ se recogieron muestras para estudiar la actividad del catalizador con el tiempo.

\section{Resultados y discusión}

Los resultados de la caracterización del catalizador por TPR mostraron que al incorporar un $25 \%$ Fe en el catalizador, Fe25, el sólido se reduce a menor temperatura $\left(553{ }^{\circ} \mathrm{C}\right)$ que sin la adicion de Fe (612 $\left.{ }^{\circ} \mathrm{C}\right)$. Por otro lado, se observó que, con la incorporación de un 25\% de Fe, la superficie específica del catalizador disminuye de 274 a 223 $\mathrm{m}^{2} / \mathrm{g}$.

Analizando los resultados tras la reacción, se vio que con un $25 \%$ de $\mathrm{Fe}$ en el catalizador se obtiene más conversión de glicerina que sin incorporar Fe en el sólido, obteniendo $50,71 \%$ y $35,93 \%$ respectivamente. También con Fe25 se obtiene más conversión de carbono a gas (6,57\% frente a 4,77\%) como a líquidos (35,46\% frente a 25,72\%). Además, favorece la producción de líquidos de valor añadido como el 1,2-propanodiol, producto de interés (ver Figura 1). Hay una pequeña variación entre los resultados de la conversión de glicerina y el total de la conversión de carbono a productos. Por debajo de un déficit de carbono inferior a $15 \%$ se considera fiable el experimento, como se plantea por otros autores [4,5].

\section{Conclusiones}

La presencia del 25\% de Fe en el catalizador favorece la conversión de glicerina, obteniendo más conversión de carbono a productos (gas y líquidos), siendo el 1,2-propanodiol el producto líquido con más selectividad.

Además, con un contenido de Fe del 25\%, el catalizador se reducía a menor temperatura que en ausencia de Fe. Sin embargo, presentaba menor superficie específica.

Tabla 1. Condiciones experimentales

\begin{tabular}{|c|c|c|c|}
\hline $\begin{array}{c}\mathrm{T} \\
\left({ }^{\circ} \mathrm{C}\right)\end{array}$ & $\begin{array}{c}\mathrm{P} \\
(\mathrm{bar})\end{array}$ & $\begin{array}{c}\mathrm{F} \\
(\% \text { peso })\end{array}$ & $\begin{array}{c}\mathrm{W} / \mathrm{m} \\
\left(\mathrm{g}_{\text {cat }} \cdot \mathrm{min} / \mathrm{g}_{\text {glicerina }}\right)\end{array}$ \\
\hline 227 & 33 & 10 & 20 \\
\hline
\end{tabular}

\section{Agradecimientos}

Los autores quieren expresar su gratitud a AEI / FEDER, UE (proyecto CTQ2017-86893-R) por el apoyo financiero recibido. También agradecen la financiación recibida del Gobierno de Aragón (Referencia T22_17R) y cofinanciado con FEDER 2014-2020 “Construyendo Europa desde Aragón”.

\section{REFERENCIAS}

[1]. LUO, X., GE, X., CUI, Sh. and LI, Y. Value-added processing of crude glycerol into chemicals and polymers. Bioresource Technology. 2016, 215, 144154. Available from: doi: 10.1016/j.biortech.2016.03.042.

[2]. ZHOU, C. H., ZHAO, H., TONG, D. S., WU L. M. and YU, W. H. Recent Advances in Catalytic Conversion of Glycerol. Science and Engineering. 2013, 55, 369453. Available from: doi: 10.1080/01614940.2013.816610.

[3]. REMÓN, J., GIMÉNEZ, J.R., VALIENTE, A., GARCÍA, L. and ARAUZO, J. Production of gaseous and liquid chemicals by aqueous phase reforming of crude glycerol: Influence of operating conditions on the process. Energy Conversion and Management. 2016, 110, 90-112. Available from: doi: 10.1016/j.enconman.2015.11.070.

[4]. GARCÍA, L., VALIENTE, A., OLIVA, M., RUIZ, J., and ARAUZO, J. Influence of operating variables on the aqueous-phase reforming of glycerol over a $\mathrm{Ni} / \mathrm{Al}$ coprecipitated catalyst. International Journal of Hydrogen Energy. 2018, 43, 20392-20407. Available from: doi: 10.1016/j.ijhydene.2018.

[5]. ROY, D., SUBRAMANIAM, B. and CHAUDHARI, R. Aqueous phase hydrogenolysis of glycerol to 1,2propanediol without external hydrogen addition. Catal Today. 2010, 156, 31-37. Available from: doi: 10.1016/j.cattod.2010.01.007.

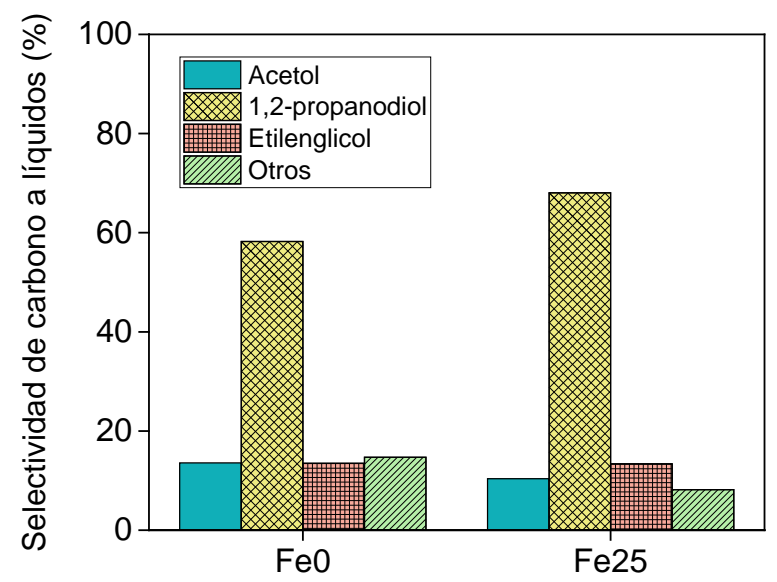

Figura 1. Selectividad de carbono a líquidos 